\title{
Adjuvant Hepatic Arterial Infusion Chemotherapy after Hepatic Resection of Hepatocellular Carcinoma With Macroscopic Vascular Invasion
}

\author{
Hidetoshi Nitta - Toru Beppu - Katsunori Imai • \\ Hiromitsu Hayashi · Akira Chikamoto • \\ Hideo Baba
}

Published online: 22 February 2013

(C) Société Internationale de Chirurgie 2013

\begin{abstract}
Background The prognosis of hepatocellular carcinoma (HCC) with macroscopic vascular invasion is extremely poor even after hepatic resection. We aimed to clarify the efficacy of adjuvant hepatic arterial infusion chemotherapy (HAI) for HCC with vascular invasion.

Methods A total of 73 HCC patients with macroscopic vascular invasion were divided into two groups: 38 with hepatectomy with HAI (HAI group) and 35 with hepatectomy alone (non-HAI group). From 1997 to 2007, HAI was performed via an implanted injection port. The treatment comprised three courses of weekly infusion of HAI, which comprised cisplatin (10 mg daily on days $1-5$ ) followed by 5-fluorouracil (5-FU; $250 \mathrm{mg}$ daily on days 1-5) infusion. From 2007 , cisplatin $\left(60 \mathrm{mg} / \mathrm{m}^{2}\right), 5-\mathrm{FU}\left(600 \mathrm{mg} / \mathrm{m}^{2}\right)$, and a mixture of mitomycin $\mathrm{C}\left(3 \mathrm{mg} / \mathrm{m}^{2}\right)$ and degradable starch microspheres were administered for two courses.

Results Overall, $92 \%$ of patients completed adjuvant HAI. In the HAI and non-HAI groups, the 5-year disease-free survival (DFS) rates were $33.1 \%$ and $11.8 \%$, respectively $(p=0.029)$, and the 5-year overall survival (OS) rates were $46.7 \%$ and $32.7 \%$, respectively $(p=0.318)$. Among the
\end{abstract}

H. Nitta - T. Beppu - K. Imai - H. Hayashi - A. Chikamoto H. Baba $(\square)$

Department of Gastroenterological Surgery, Graduate School of Medical Sciences, Medical School Hospital, Kumamoto University, 1-1-1 Honjo, Kumamoto 860-8556, Japan

e-mail: hdobaba@kumamoto-u.ac.jp

H. Nitta

e-mail: hnitta5085@gmail.com

T. Beppu

Department of Multidisciplinary Treatment for

Gastroenterological Cancer, Innovation Center for Translational

Research, Kumamoto University Hospital, Kumamoto, Japan patients with $\mathrm{Vp} 3 / 4$ or $\mathrm{Vv} 3(n=32)$ in the HAI group, the 3-year DFS and OS rates were $33.7 \%$ and $56.8 \%$, respectively $(p=0.049)$. Those in the non-HAI group were $8.3 \%$ and $12.0 \%$, respectively $(p=0.023)$. Cox proportional multivariate analysis for DFS revealed that HAI was an independent favorable prognostic factor in all 73 patients (hazard ratio $0.536 ; p=0.029$ ).

Conclusions Adjuvant HAI for HCC patients with vascular invasion might reduce the risk of recurrence.

\section{Introduction}

Hepatocellular carcinoma (HCC) is a common malignancy worldwide [1]. Hepatectomy achieves favorable outcomes in well-selected candidates and is still considered one of the most potentially curative treatments for HCC [2]. Unfortunately, the long-term survival after hepatectomy is unsatisfactory because of the high incidence of tumor recurrence, especially intrahepatic tumors [3]. After curative hepatic resection for HCC, the recurrence rates at 2 and 5 years are approximately $50 \%$ to $60 \%$ and $80 \%$, respectively [4-6]. The prognosis remains extremely poor in those with advanced HCC with distinct vascular invasion, such as tumor thrombosis of the first branch or trunk of the portal vein or inferior vena cava [7, 8]. Portal vein tumor thrombosis (PVTT) can cause widespread dissemination of tumor cells via the portal tract as well as liver dysfunction and portal vein hypertension. In turn, this can lead to intractable ascites, variceal rupture, hepatic encephalopathy, and/or death [9]. The risks of PVTT with intrahepatic dissemination after local ablation therapye.g., ethanol injection therapy, microwave coagulation therapy, radiofrequency ablation-for small HCCs adjacent to the main or sectional portal vein were recently 
reported [10]. However, even with the removal of a macroscopic PVTT, there may still be microscopic tumor thrombi, which can be the source of a recurrence. Thus, an effective adjuvant therapy is required to prevent tumor recurrence after hepatic resection treatments for patients with vascular invasion.

Recent advances in implantable drug delivery systems have facilitated repeated hepatic arterial infusion of chemotherapy (HAI). Several studies report that intraarterial 5-fluorouracil (5-FU) with low-dose cisplatin [7, 11, 12] or 5-FU and systemic interferon (IFN $\alpha$ ) [13] are the most effective combinations for unresectable HCCs with PVTT. However, there is little convincing evidence indicating that adjuvant therapy reduces the risk of recurrence after hepatic resection. Furthermore, no standard regimen has been established.

In the present study, we assessed the efficacy and feasibility of intraarterial cisplatin/5-FU combination therapy for surgically resected HCCs with massive vascular invasion. We identified patients subsets who would most likely benefit from adjuvant HAI.

\section{Methods}

From April 1997 to March 2011, a total of 539 patients underwent hepatic resection for $\mathrm{HCC}$ at Kumamoto University Hospital. Among them, the 77 patients who underwent resection of $\mathrm{HCC}$ with macroscopic vascular invasion $(\geq \mathrm{Vp} 2$ or $\geq \mathrm{Vv} 2$ ) and without distant metastasis were enrolled in this study. The invasion sites of portal venous invasion ( $\mathrm{Vp}$ ) and hepatic venous invasion ( $\mathrm{Vv}$ ) were defined as follows: $\mathrm{Vp} 2$, second-order branches of the portal vein; $\mathrm{Vp} 3$, first-order branches of the portal vein; Vp4, main trunk or opposite the first branch of the portal vein; $\mathrm{Vv} 2$, main trunk of the hepatic vein; Vv3, inferior vena cava. Portal or hepatic venous invasion was diagnosed based on the findings of preoperative imaging studies, such as dynamic computed tomography (CT) or magnetic resonance imaging (MRI) and CT angiography. The diagnosis of HCC was confirmed by histopathological examinations of the resected specimens.

The entry criteria of HAI group were as follows: no recurrence confirmed 1 month after hepatic resection; appropriate liver function reserve; good performance status; sufficient recovery from the operation. Four patients died because of postoperative complications (liver failure 2; acute respiratory distress syndrome 1 ; intraabdominal infection 1 ) and were excluded from analysis. The remaining 73 patients were reviewed retrospectively. In all, 38 patients treated with hepatic resection and HAI were allocated to the HAI group. During the same period, 35 patients who underwent hepatic resection without HAI therapy by the same surgical team were allocated to the non-HAI group. The reasons why patients did not receive HAI were as follows: early recurrence $(n=10)$, disapproval of the therapy $(n=10)$, advanced age $(n=4)$, co-morbidities ( $n=7)$, poor performance status $(n=4)$.

Patients diagnosed with multiple and hypervascular tumor recurrence in the remnant liver on CT angiography 1 month after hepatic resection $(n=10)$ were treated with transarterial chemoembolization (TACE) using cisplatin suspended in Lipiodol with a gelatin sponge instead of HAI according to our treatment strategy [14]. This study was conducted in accordance with the Declaration of Helsinki and the ethical guidelines for clinical studies of the Ministry of Health, Labor, and Welfare in Japan. Written informed consent was obtained from all patients.

\section{Surgical technique}

All patients underwent hepatic resection performed by two senior liver surgeons. The operative procedure was determined beforehand on the basis of the liver function reserve, the extent of the main and satellite tumors, and portal or venous invasion. Parenchymal dissection was performed using an ultrasonic surgical aspirator (CUSA; Valley Lab, Boulder, CO, USA) and bipolar forceps with intermittent clamping of the portal triad. After 2005, we preferred a precoagulation technique using a dissecting sealer (Valley $\mathrm{Lab}$ ) or the VIO soft coagulation system (ERBE, Elektromedizin GmbH, Tübingen, Germany) [15]. For the patients with Vp4, the PVTT was removed using the "peeling off" technique [16]. In brief, the portal venotomy was placed after adequate vascular control of the portal flow was established. The PVTT was dissected from the portal venous wall and removed via the opening. Macroscopic residual PVTTs intruding into tiny branches were extracted meticulously. A multiperforated drain was placed in the abdominal cavity at the end of the procedure.

\section{HAI treatment}

From 1997 to 2007, patients with HCCs were treated by arterial infusion of a chemotherapeutic agent via a subcutaneously implanted injection port (old protocol). In principle, a hepatic arterial catheter was placed 2 to 3 weeks after the operation via the femoral artery. Celiac angiography was performed according to the Seldinger technique. A 4For 5F heparin-coated catheter was introduced into the proper or common hepatic artery. The gastroduodenal and right gastric arteries were occluded using a steel coil to prevent gastroduodenal injury caused by anticancer agents. After the catheter was connected to the injection port, the device was implanted in a subcutaneous pocket in the right lower quadrant to avoid catheter kinks. One course consisted of cisplatin administration (10 mg daily on days 1-5) and subsequent infusion of 5-FU (250 mg daily on days 1-5). After confirming a lack of recurrence in the remnant 
liver by CT angiography 4 weeks after surgery, HAI was started and repeated for 3 courses. The injection port was removed 1 week after the end of HAI with confirmation of a lack of recurrence.

From 2007, patients with HCC were treated without an injection port (new protocol). Briefly, a $4 \mathrm{~F}$ or $5 \mathrm{~F}$ heparincoated catheter was introduced into the proper hepatic artery. Cisplatin $\left(60 \mathrm{mg} / \mathrm{m}^{2}\right)$ dissolved in $100 \mathrm{ml}$ saline for $10 \mathrm{~min}$ followed by 5 -FU $\left(600 \mathrm{mg} / \mathrm{m}^{2}\right)$ in $100 \mathrm{ml}$ saline for $10 \mathrm{~min}$ were injected into the proper and more distant hepatic arteries, respectively. Then, mitomycin $\mathrm{C}\left(3 \mathrm{mg} / \mathrm{m}^{2}\right)$ dissolved in 3 to $5 \mathrm{ml}$ of saline mixed with 3 to $5 \mathrm{ml}$ of degradable starch microspheres (DSMs) (Spherex; Yakult, Tokyo, Japan) was administered. This procedure was repeated twice at 4 weeks after surgery with a 1-month interval.

Follow-up

The follow-up program included serum $\alpha$-fetoprotein (AFP) and protein induced by vitamin $\mathrm{K}$ absence or antagonists-II (PIVKA-II) assays every 1 to 2 months. Imaging follow-up was performed using transabdominal ultrasonography (US) examination or computed tomography (CT) every 3 to 4 months. Magnetic resonance imaging (MRI) was performed when deemed necessary. During follow-up, if recurrence was recognized the patient was treated with a second hepatectomy, TACE, radiofrequency ablation, radiotherapy, or chemotherapy.

Data collection

Information on 15 variables was collected for each patient as potential risk factors for recurrence and predictors of survival. The extents of vascular involvement and tumor differentiation were confirmed in the resected specimen. Segmentectomy of $\geq 3$ was defined as a major hepatic resection. Others were defined as minor hepatic resections. The continuous variables of age, indocyanine green retention at 15 min (ICG-R15), main tumor size, and serum AFP and PIVKA-II levels were categorized by cutoff values according to their median values: 64 years, $10 \%$, $60 \mathrm{~mm}, 70.2 \mathrm{ng} / \mathrm{ml}$, and $697 \mathrm{IU} / \mathrm{ml}$, respectively.

\section{Statistical analyses}

Student's $t$ test, the $\chi^{2}$ test,or Fisher's exact test was used where appropriate to compare the clinical and histologic parameters between the two groups. The cumulative survival curves were obtained using the Kaplan-Meier method. Survival curves were statistically compared using the log-rank test. Univariate analysis of the data from all cases was performed. Variables that exhibited statistical significance in the univariate analysis were subsequently included in the multivariate analysis, which was performed using Cox proportional hazard analysis. For all tests, the level of significance was set at $p<0.05$.

\section{Results}

The clinical and pathological characteristics of the patients are summarized in Tables 1 and 2. The mean age was significantly lower in the HAI group than in the non-HAI group. The rate of poor differentiation was significantly higher in the HAI group than in the non-HAI group. However, there were no significant differences between the
Table 1 Clinical profile and serologic assays for HCC patients who underwent surgery: HAI group versus nonHAI group

HCC hepatocellular carcinoma, $H A I$ hepatic arterial infusion chemotherapy, $H B s-A g$ hepatitis $\mathrm{B}$ surface antigen, $H C V-A b$ hepatitis $\mathrm{C}$ virus antibodies, $I C G-R 15$ indocyanine green retention rate at $15 \mathrm{~min}, A F P \alpha$ fetoprotein, PIVKA-II protein induced by vitamin $\mathrm{K}$ absence or antagonists II

a Including the following: $\mathrm{HBsAg}^{+} / \mathrm{HCVAb}^{+}$; both positive; both negative

\begin{tabular}{llll}
\hline Parameter & $\begin{array}{l}\text { HAI group } \\
(n=38)\end{array}$ & $\begin{array}{l}\text { Non-HAI group } \\
(n=35)\end{array}$ & $p$ \\
\hline Age (years) & $61.8 \pm 8.8$ & $65.7 \pm 8.8$ & 0.036 \\
Sex $(\mathrm{M} / \mathrm{F})$ & $34 / 4$ & $28 / 7$ & $\mathrm{NS}$ \\
Hepatitis $^{\mathrm{a}}$ & $16 / 13 / 0 / 9$ & $7 / 21 / 1 / 6$ & $\mathrm{NS}$ \\
Albumin $(\mathrm{g} / \mathrm{dl})$ & $4.0 \pm 0.5$ & $3.8 \pm 0.4$ & $\mathrm{NS}$ \\
Total bilirubin $(\mathrm{mg} / \mathrm{dl})$ & $0.75 \pm 0.2$ & $0.77 \pm 0.2$ & $\mathrm{NS}$ \\
Prothrombin activity $(\%)$ & $90.8 \pm 13.5$ & $95.1 \pm 11.9$ & $\mathrm{NS}$ \\
Platelet count $\left(\times 10^{4} / \mu \mathrm{l}\right)$ & $13.4 \pm 7.3$ & $15.3 \pm 12.5$ & $\mathrm{NS}$ \\
Child Pugh score $(\mathrm{A} / \mathrm{B})$ & $38 / 0$ & $34 / 1$ & $\mathrm{NS}$ \\
ICG-R15 $\%)$ & $13.4 \pm 7.3$ & $15.3 \pm 12.5$ & $\mathrm{NS}$ \\
AFP $(\mathrm{ng} / \mathrm{ml})$ & $97.6(3-292,829)$ & $55.5(1.6-474,000)$ & $\mathrm{NS}$ \\
AFP-L3 $(\geq 10 \% /<10 \%)$ & $15 / 23$ & $17 / 18$ & $\mathrm{NS}$ \\
PIVKA-II $(\mathrm{mAU} / \mathrm{ml})$ & $504(11-18574)$ & $1211(0-298,050)$ & $\mathrm{NS}$ \\
Tumor size $(\mathrm{mm})$ & $66.6 \pm 39.3$ & $70.0 \pm 42.3$ & $\mathrm{NS}$ \\
Multiple tumors $(\mathrm{yes} / \mathrm{no})$ & $25 / 13$ & $23 / 12$ & $\mathrm{NS}$ \\
\hline
\end{tabular}


Table 2 Perioperative results and histologic findings for $\mathrm{HCC}$ patients who underwent surgery: HAI group versus nonHAI group

UICC stage: International Union against Cancer staging

\begin{tabular}{llll}
\hline Parameter & $\begin{array}{l}\text { HAI group } \\
(n=38)\end{array}$ & $\begin{array}{l}\text { Non-HAI group } \\
(n=35)\end{array}$ & $p$ \\
\hline Operating time (min) & $492 \pm 131$ & $481 \pm 105$ & NS \\
Blood loss (ml) & $1079 \pm 882$ & $1009 \pm 965$ & NS \\
Blood transfusion ( \pm ) & $14 / 24$ & $11 / 24$ & NS \\
Type of resection (major/minor) & $30 / 8$ & $26 / 9$ & NS \\
UICC stage 2/3A/3B/4A & $3 / 8 / 25 / 2$ & $4 / 10 / 19 / 2$ & NS \\
Tumor differentiation (well to moderate/poor/unknown) & $14 / 23 / 1$ & $22 / 12 / 1$ & 0.023 \\
Tumor type (simple nodular/others) & $21 / 17$ & $15 / 20$ & NS \\
Surgical margin (干) & $33 / 5$ & $32 / 3$ & NS \\
Portal venous invasion (Vp0 1/2/3/4) & $7 / 14 / 14 / 3$ & $8 / 16 / 9 / 2$ & NS \\
Hepatic venous invasion (Vv0 1/2/3) & $26 / 11 / 1$ & $21 / 12 / 2$ & NS \\
\hline
\end{tabular}

HAI and non-HAI groups with respect to any other variable. The surgical parameters including operative procedure, operative duration, and blood infusion rates, were comparable between the two groups.

Compliance and side effects of chemotherapy and hepatic resection

A total of 35 patients (92\%) completed HAI after hepatic resection. The side effects of adjuvant HAI (according to CTCAE v4.0) are shown in Table 3 .HAI was stopped because of tumor recurrence, grade 4 neutropenia, and therapy refusal in one patient each. No lethal side effects of HAI were observed. Five patients $(13 \%)$ experienced grade $3 / 4$ adverse events. Three patients had grade 3 vomiting, and three had severe neutropenia (one with grade 4 , two with grade 3 ). One patient $(4.1 \%)$ developed a hepatic arterial occlusion caused by the implanted catheter. Among all 73 patients, postoperative complications occurred in $21(28.7 \%)$ : significant pleural effusion or ascites in 7 , bile leakage in 3, surgical-site infection in 3 , liver failure in 2 , abdominal abscess in 2 , intraabdominal bleeding in 1 , duodenal ulcer in 1 , wound dehiscence in 1 , and cholangitis in 1 .

Table 3 Side effects of adjuvant HAI $(n=38)$

\begin{tabular}{lll}
\hline Side effect & All & Grade3/4 \\
\hline Neutropenia & $9(23.7 \%)$ & $3(7.9 \%)$ \\
Thrombocytopenia & $3(7.9 \%)$ & 0 \\
Vomiting & $8(21.0 \%)$ & $3(7.9 \%)$ \\
Poor appetite & $5(13.1 \%)$ & 0 \\
Ascites & $1(2.6 \%)$ & 0 \\
Acute kidney injury & $1(2.6 \%)$ & 0 \\
Bilirubin & $5(13.1 \%)$ & 0 \\
Hepatic arterial occlusion & $1(2.6 \%)$ & - \\
\hline
\end{tabular}

Tumor recurrence

At a median follow-up of 25 months (range 3-149 months), $23(60.5 \%)$ and $29(82.8 \%)$ patients in the HAI and nonHAI groups, respectively, developed tumor recurrence. The rate of recurrence in the remnant liver in the HAI group was $31.5 \%$, which was significantly lower than $68.5 \%$ in the non-HAI group ( $p=0.001)$. Extrahepatic recurrence rates were not significantly different between the two groups $(p=0.360)$. In all, 45 patients-20 (52.6\%) in the HAI group and $25(71.4 \%)$ in the non-HAI group-developed recurrence within 2 years after hepatic resection, but the recurrence rates within 2 years were similar between the two groups $(p=0.098)$.

Disease-free and overall survival

There was no significant difference in recurrence or survival rates between the patients who received HAI according to the old $(n=25)$ and new $(n=13)$ protocols: 3-year diseasefree survival (DFS) $55.0 \%$ vs. $50.0 \%$; 3-year overall survival (OS), $53.0 \%$ vs. $54.2 \%$ ). Both the 3- and 5-year DFS rates were $33.1 \%$ for patients treated with HAI and $11.8 \%$ for patients in the non-HAI group. The HAI group achieved significantly better outcomes than the non-HAI group ( $p=0.029$ ) (Fig. 1a). The 3- and 5-year OS rates were $56.2 \%$ and $46.7 \%$ in the HAI group and $37.4 \%$ and $32.7 \%$ in the non-HAI group, respectively ( $p=0.318$ ) (Fig. 1b).

Univariate analysis for DFS and OS was performed for the 73 patients with macroscopic vascular invasion. Tumor size, number of tumors, International Union Against Cancer stage (UICC, 7th edition), and HAI were recognized as potential prognostic factors for DFS (Table 4). AFP, tumor size, and the degree of vascular invasion were recognized as potential prognostic factors for OS (Table 5). Multivariate analysis revealed that the number of tumors [hazard ratio $(\mathrm{HR})$ and $95 \%$ confidence interval (CI): 2.246 (1.158-4.359)] and HAI [HR and $95 \%$ CI): 0.536 (0.306-0.940)] were 

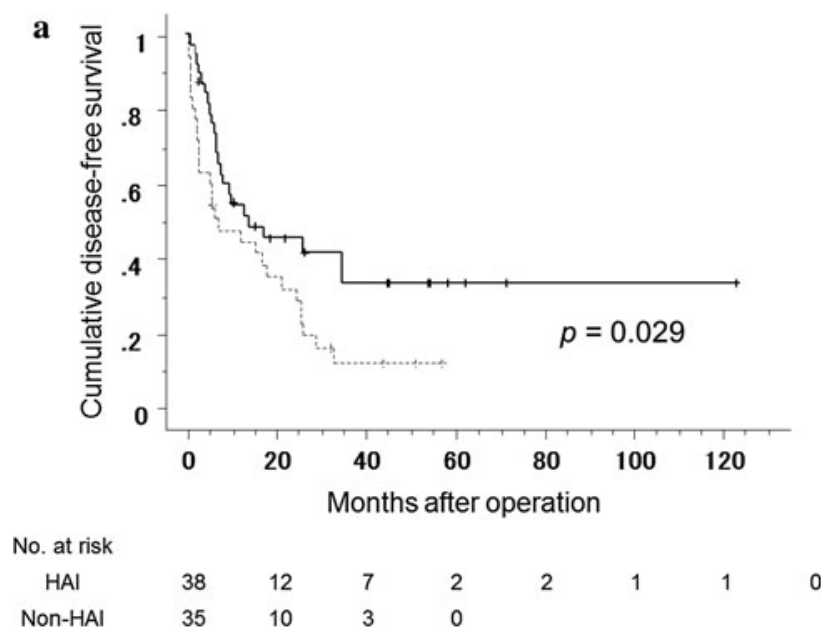

b

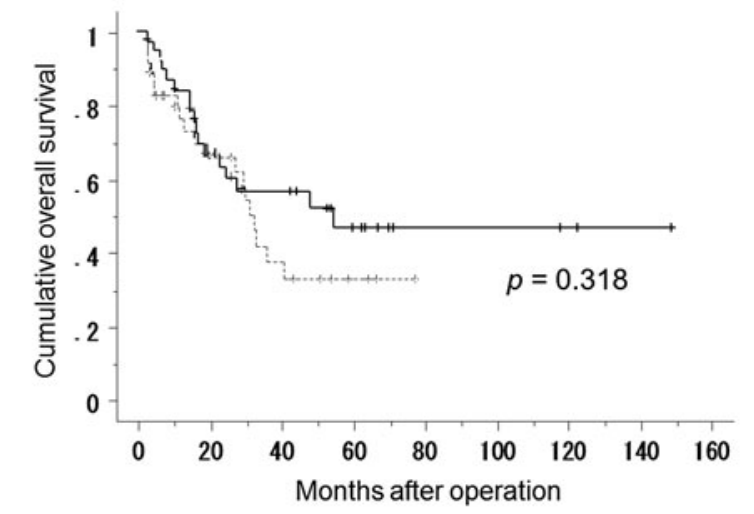

No. at risk

$\begin{array}{ccccccccc}\text { HAI } & 38 & 21 & 15 & 9 & 3 & 3 & 2 & 0 \\ \text { Non-HAl } & 35 & 19 & 8 & 3 & 0 & & & \end{array}$

Fig. 1 a Disease-free survival (DFS) curves after hepatic resection with or without hepatic arterial infusion (HAI) chemotherapy in 73 patients. The HAI group (solid line) exhibited significantly better DFS than the non-HAI group (dashed line) $(p=0.029)$. b Overall survival (OS) curves after hepatic resection in the HAI (solid line) and the non-HAI groups (dashed line) in 73 patients. There was no significant difference between the two groups with respect to OS $(p=0.318)$

independent factors for DFS (Table 6). Only tumor size of $\geq 60 \mathrm{~mm}$ was an independent prognostic factor for OS in the multivariate analysis ratio [HR and $95 \%$ CI: 2.296 (1.106-4.767); $p=0.025]$.

On the other hand, among the limited patients with $\mathrm{Vp} 3 / 4$ or Vv3 $(n=32)$, the 3-year DFS and OS rates were $33.7 \%$ and $56.8 \%$ in the HAI group and $8.3 \%$ and $12.0 \%$ in the non-HAI group, respectively (Fig. 2). The HAI group had significantly better DFS and OS than the non-HAI group $(p=0.023$ and 0.049 , respectively). However, among patients with Vp2 or Vv2, the 3-year DFS and OS rates were $32.6 \%$ and $57.0 \%$ in the HAI group and $13.7 \%$ and $52.0 \%$ in the non-HAI group, respectively. There were no significant differences between these two groups.
Table 4 Univariate analysis for disease-free survival $(n=73)$

\begin{tabular}{|c|c|c|}
\hline Factor & Median DFS (months) & $p$ \\
\hline Age (years) & & 0.770 \\
\hline$<64$ & 9.5 & \\
\hline$\geq 64$ & 15.4 & \\
\hline Sex & & 0.961 \\
\hline Male & 17.4 & \\
\hline Female & 27.2 & \\
\hline Tumor size (mm) & & 0.035 \\
\hline$<60$ & 18.0 & \\
\hline$\geq 60$ & 6.4 & \\
\hline Tumor number & & 0.006 \\
\hline Solitary & 33.0 & \\
\hline Multiple & 7.9 & \\
\hline Operative method & & 0.309 \\
\hline Minor & 16.8 & \\
\hline Major & 9.5 & \\
\hline ICG-R15 (\%) & & 0.431 \\
\hline$<10$ & 12.3 & \\
\hline$\geq 10$ & 15.4 & \\
\hline AFP (ng/ml) & & 0.704 \\
\hline$<70.2$ & 13.0 & \\
\hline$\geq 70.2$ & 15.4 & \\
\hline PIVKA-II (mAU/ml) & & 0.105 \\
\hline$<697$ & 17.3 & \\
\hline$\geq 697$ & 6.5 & \\
\hline UICC stage & & 0.002 \\
\hline 2 or $3 \mathrm{~A}$ & 6.5 & \\
\hline $3 \mathrm{~B}$ or 4 & 33.0 & \\
\hline Tumor differentiation & & 0.816 \\
\hline Well to moderately & 13.0 & \\
\hline Poor & 8.4 & \\
\hline Vascular invasion & & 0.349 \\
\hline $\mathrm{Vp} 2, \mathrm{Vv} 2$ & 14.0 & \\
\hline Vp3-4, Vv3 & 6.5 & \\
\hline Tumor type & & 0.199 \\
\hline Simple nodular & 24.7 & \\
\hline Others & 9.5 & \\
\hline Blood transfusion & & 0.176 \\
\hline Yes & 6.4 & \\
\hline No & 16.8 & \\
\hline Stage of fibrosis ${ }^{a}$ & & 0.290 \\
\hline F0-II & 24.7 & \\
\hline FIII-IV & 7.9 & \\
\hline HAI & & 0.029 \\
\hline Yes & 14.0 & \\
\hline No & 7.2 & \\
\hline
\end{tabular}

DFS disease-free survival

${ }^{a}$ New Inuyama classification [35] 
Table 5 Univariate analysis for OS $(n=73)$

\begin{tabular}{|c|c|c|}
\hline Factors & Median survival (months) & $p$ \\
\hline Age (years) & & 0.972 \\
\hline$<64$ & 33.3 & \\
\hline$\geq 64$ & 33.0 & \\
\hline Sex & & 0.961 \\
\hline Male & 17.4 & \\
\hline Female & 27.2 & \\
\hline Tumor size $(\mathrm{mm})$ & & 0.023 \\
\hline$<60$ & - & \\
\hline$\geq 60$ & 27.2 & \\
\hline Tumor number & & 0.067 \\
\hline Solitary & - & \\
\hline Multiple & 30.0 & \\
\hline Operative method & & 0.919 \\
\hline Minor & 31.6 & \\
\hline Major & 33.3 & \\
\hline ICG-R15 (\%) & & 0.473 \\
\hline$<10$ & 13.3 & \\
\hline$\geq 10$ & 19.7 & \\
\hline $\mathrm{AFP}(\mathrm{ng} / \mathrm{mL})$ & & 0.032 \\
\hline$<70.2$ & - & \\
\hline$\geq 70.2$ & 33.0 & \\
\hline PIVKA-II (mAU/ml) & & 0.080 \\
\hline$<697$ & 54.8 & \\
\hline$\geq 697$ & 27.2 & \\
\hline UICC stage & & 0.880 \\
\hline 2 or $3 \mathrm{~A}$ & 36.4 & \\
\hline $3 \mathrm{~B}$ or 4 & 33.0 & \\
\hline Tumor differentiation & & 0.761 \\
\hline Well to moderately & 33.3 & \\
\hline Poor & 54.8 & \\
\hline Vascular invasion & & 0.025 \\
\hline $\mathrm{Vp} 2, \mathrm{Vv} 2$ & 48.3 & \\
\hline Vp3-4, Vv3 & 19.7 & \\
\hline Tumor type & & 0.689 \\
\hline Simple nodular & 54.8 & \\
\hline Others & 33.0 & \\
\hline Blood transfusion & & 0.088 \\
\hline Yes & 24.8 & \\
\hline No & 41.3 & \\
\hline Stage of fibrosis ${ }^{a}$ & & 0.256 \\
\hline F0-II & 48.3 & \\
\hline FIII-IV & 31.6 & \\
\hline HAI & & 0.318 \\
\hline Yes & 54.8 & \\
\hline No & 31.6 & \\
\hline
\end{tabular}

${ }^{a}$ New Inuyama classification [35]
Table 6 Multivariate analysis for DFS

\begin{tabular}{llll}
\hline Factor & $p$ & HR & $95 \%$ CI \\
\hline Tumor size & 0.091 & 1.623 & $0.925-2.849$ \\
Multiple tumors & 0.017 & 2.246 & $1.158-4.359$ \\
HAI & 0.029 & 0.536 & $0.306-0.940$ \\
\hline
\end{tabular}

HR: hazard ratio; CI: confidence interval
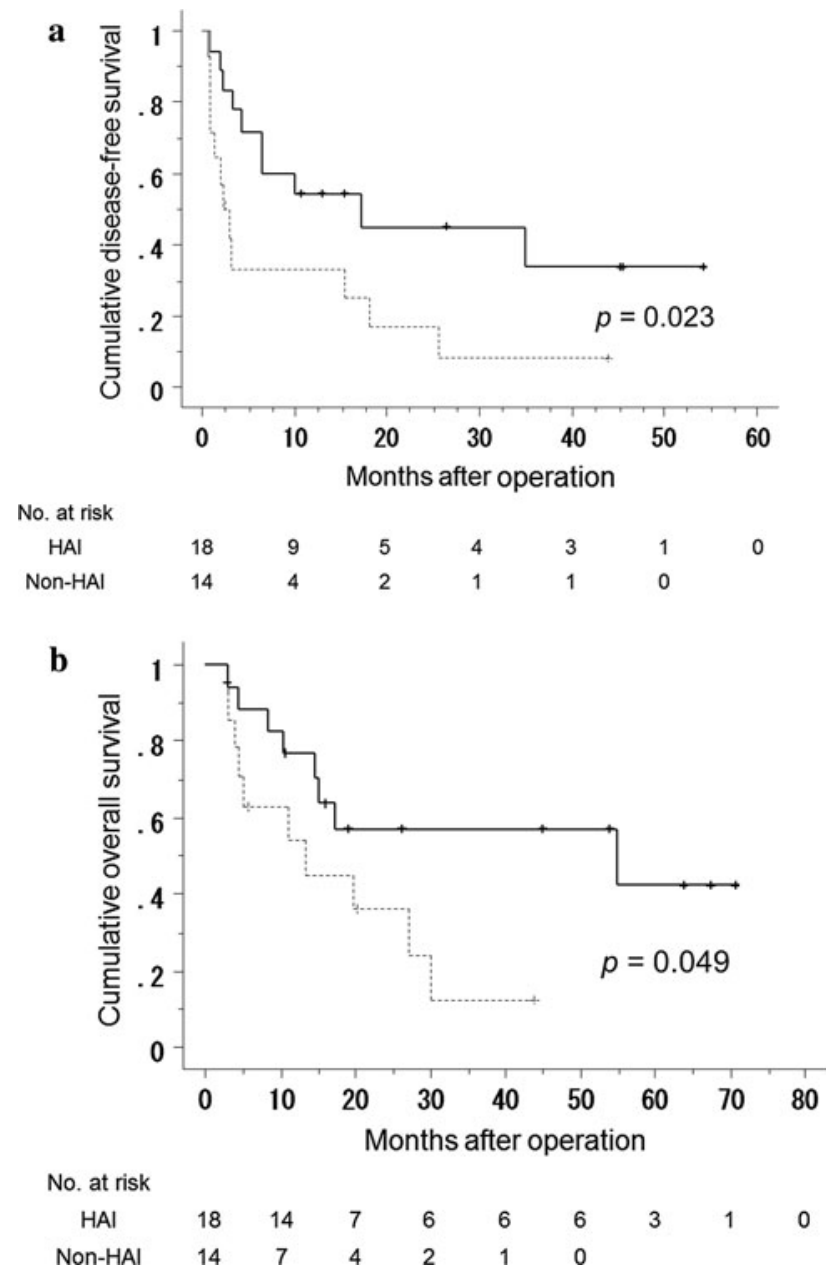

Fig. 2 a DFS curves after hepatic resection with or without HAI for the patients with $\mathrm{Vp} 3 / 4$ or $\mathrm{Vv} 3(n=32)$. The HAI group (solid line) showed significantly better DFS than the non-HAI group (dashed line) $(p=0.023)$. b OS curves after hepatic resection with or without HAI for the patients with $\mathrm{Vp} 3 / 4$ or $\mathrm{Vv} 3(n=32)$. The HAI group (solid line) showed significantly better OS than the non-HAI group (dashed line) $(p=0.049)$

\section{Discussion}

The prognosis of HCC patients with apparent PVTT is limited to a few months after diagnosis [8, 17-19]. Although hepatic resection as a monotherapy demonstrates 
relatively favorable 5-year survival rates (4.0-28.5\%) and median survival times (6-14 months), the recurrence rates are extremely high [20-24]. Portal venous invasion is the most significant risk factor for the early postoperative recurrence of HCC [25]. Therefore, other novel strategies to prevent recurrence are required. At present, combined treatment consisting of hepatic resection and TACE or HAI shows the most promising results. The 5-year survival rate and median survival time for TACE followed by hepatic resection are $42 \%$ and 31 months, respectively [26]. For hepatic resection followed by HAI, these numbers are $36 \%$ and 22 months. respectively [11].

Accordingly, in 1997 we initially started the protocol of arterial infusion of cisplatin (total $150 \mathrm{mg}$ ) followed by 5-FU (total $3750 \mathrm{mg}$ ). The rationale behind this treatment regimen is that cisplatin and 5-FU have an antitumor effect. Moreover, cisplatin has a synergistic effect as a modulator for 5-FU, and cisplatin and 5-FU can be administered at low doses to reduce adverse reactions. The treatment duration of our protocol-three courses of low-dose cisplatin plus 5-FU-is shorter than that reported in the literature. However, it may be sufficient to reduce the risk of recurrence because of the favorable prognosis of our treatment. The 3-year DFS and OS were $55.0 \%$ and $53.0 \%$, respectively, which are comparable to those reported in the literature [27-29]. Nevertheless, one patient developed hepatic arterial occlusion caused by the implanted catheter.

Based on this experience, in 2007 we initiated a new protocol without an injection port: two rounds of one-shot HAI consisting of cisplatin $\left(60 \mathrm{mg} / \mathrm{mm}^{2}\right.$ per $\left.10 \mathrm{~min}\right), 5-\mathrm{FU}$ $\left(60 \mathrm{mg} / \mathrm{mm}^{2}\right.$ per $\left.10 \mathrm{~min}\right)$ followed by mitomycin-C $(3 \mathrm{mg} /$ $\mathrm{mm}^{2}$ ) with DSM. In the new protocol, we used arterial administration using DSM and mitomycin-C. DSM is a micro-embolic material made from potato starch, $45 \pm 7 \times 10^{-6} \mathrm{~mm}$ in diameter, that induces transient occlusion of small arteries for $1 \mathrm{~h}$ [30]. Co-administration of an anticancer drug with DSM reduces drug dilution because of hepatic arterial flow and enhances drug retention in tumors better than with a single bolus injection [30]. We adopted DSM rather than permanent embolic materials to decrease the damage to the remnant liver during the early postoperative period (4-8 weeks after surgery).

A retrospective case-control study of 127 HCC patients demonstrated that adjuvant chemolipiodolization following hepatic resection is an independent prognostic factor for 2-year recurrence-free survival [HR and $95 \% \mathrm{CI}: 0.55$ $(0.34-0.90) ; p=0.02]$ compared to hepatic resection alone [3]. However, the ratio of vascular involvement patients in that study was only $19 \%$ [3]. In a randomized controlled manner, $126 \mathrm{HCC}$ patients with PVTT were divided into control and TACE groups [27]. The control group underwent surgery and PVTT removal, and the TACE group underwent the same procedure combined with postoperative adjuvant TACE. The median survival time of 13 months and a 5-year survival rate of $21.5 \%$ in the TACE group were significantly greater than the corresponding values of 9 months and $8.5 \%$ in the control group [27]. The treatment was started 3 to 4 weeks after surgery and was repeated once every 1 to 2 months for two to five courses (mean 1.8). This postoperative adjuvant TACE resulted in significantly greater OS. The starting point and total course of adjuvant therapy were similar to those of our new protocol during the late period (beginning at 4 weeks for a total of two courses).

Despite the greater number of patients with poorly differentiated HCCs, which is associated with an extremely high recurrence rate [31], the HAI group exhibited significantly better DFS than the non-HAI group. Furthermore, multivariate analysis revealed that the application of HAI is an independent favorable prognostic factor for DFS (Fig. 1a, Table 6). On the other hand, analysis of all patients revealed comparable extrahepatic recurrence rates in the two groups and that HAI was not a favorable prognostic factor for OS. Therefore, some systemic chemotherapy in addition to the HAI therapy might be required to reduce the risk of extrahepatic recurrence, ultimately increasing the OS. Because intrahepatic metastases after hepatic resection are reported to occur usually within 2 years postoperatively [32,33] and PVTT often causes extensive intrahepatic metastasis of the tumor through the portal tract, the primary objective of adjuvant $\mathrm{HAI}$ is to reduce the risk of intrahepatic metastasis via the portal vein rather than multicentric recurrence [34]. Our results did not indicate any obvious differences between the two groups with respect to the recurrence rate within 2 years. However, the overall recurrence rate of the remnant liver in the HAI group was $31.5 \%$, which was significantly lower than the $68.5 \%$ in the non-HAI group $(p=0.001)$.

In our analysis, the HAI group demonstrated significantly better DFS and OS than the non-HAI group in the limited Vp3/4 or Vv3 patients (Fig. 2) but not in the Vp2 or $\mathrm{Vv2}$ patients. There is little information regarding recurrence or long-term survival with respect to the degree of tumor thrombi. Vp2 or Vv2 patients may not have widespread dissemination of tumor cells via the portal or hepatic vein. Furthermore, the extent of resected liver is limited, and the removal of tumor thrombi is easier and more complete than in $\mathrm{Vp} 3 / 4$ and $\mathrm{Vv} 3$ patients. In fact, operating time, blood loss, and the frequency of blood transfusion were significantly smaller in $\mathrm{Vp} 2$ and $\mathrm{Vv} 2$ patients (data not shown).

PVTT with multinodular or diffuse recurrence (i.e., intrahepatic dissemination) of HCC after thermal ablation is one of the most serious problems resulting in a poor 
prognosis [10]. In this study, seven patients exhibited this recurrence pattern according to the histopathologic examinations of the resected specimens, and five patients underwent adjuvant HAI. The 5-year OS and DFS rates of these five patients were $80 \%$ and $40 \%$, respectively, suggesting that hepatic resection followed by adjuvant HAI is also useful for PVTT with intrahepatic dissemination if the recurrence site is localized in the liver.

We applied mono- or bi-sectionectomy or (extended) hemi-hepatectomy in $42(93.3 \%)$ HCC patients with grade $\mathrm{Vp} 2$ or Vv2. Tumor thrombi might be completely removed by performing surgery alone in such cases. Among the nine patients with Vp3/4 (32.1\%), we applied the peeling-off technique for those with massive PVTTs beyond the bifurcation or into other sectors. This method is reported to have no disadvantages regarding curability compared to an en bloc technique [15]. The favorable 3-year DFS (32.4\%) and OS $(58.3 \%)$ rates of these nine patients may demonstrate the efficacy of the peeling-off technique for patients with grade $\mathrm{Vp} 3 / 4$.

Multivariate analysis revealed that adjuvant HAI was one of the independent favorable prognostic factors for DFS. However, the main limitation of this study is that it was not a randomized prospective study. Therefore, some confounders might have affected the results. The patients diagnosed with early recurrence (within 1 month) were analyzed as the non-HAI group because we aimed to investigate the advantages of adjuvant $\mathrm{HAI}$ in patients with resectable HCC and if HAI could be completed within 1 to 2 months. However, with the entry criteria used for the non-HAI group (early recurrence, advanced age, co-morbidities, poor performance), there is clearly a selection bias with less favorable prognostic factors in these patients.

\section{Conclusions}

Adjuvant HAI for HCC patients with macroscopic vascular invasion, especially patients with $\mathrm{Vp} 3 / 4$ or $\mathrm{Vv} 3$, might reduce the risk of recurrence without serious complications. However, a prospective randomized study is required to confirm our findings.

Conflict of interest The authors declare no conflicts of interest.

\section{References}

1. El-Serag HB, Mason AC (1999) Rising incidence of hepatocellular carcinoma in the United States. N Engl J Med 340:745-750

2. Poon D, Anderson BO, Chen LT et al (2009) Management of hepatocellular carcinoma in Asia: consensus statement from the Asian Oncology Summit 2009. Lancet Oncol 10:1111-1118

3. Ueno M, Uchiyama K, Ozawa S et al (2011) Adjuvant chemolipiodolization reduces early recurrence derived from intrahepatic metastasis of hepatocellular carcinoma after hepatectomy. Ann Surg Oncol 8:3624-3631

4. Imamura H, Matsuyama Y, Tanaka E et al (2003) Risk factors contributing to early and late phase intrahepatic recurrence of hepatocellular carcinoma after hepatectomy. J Hepatol 38:200-207

5. Llovet JM, Fuster J, Bruix J (1999) Intention-to-treat analysis of surgical treatment for early hepatocellular carcinoma: resection versus transplantation. Hepatology 30:1434-1440

6. Poon RT, Fan ST, Ng IO et al (2000) Different risk factors and prognosis for early and late intrahepatic recurrence after resection of hepatocellular carcinoma. Cancer 89:500-507

7. Ando E, Yamashita F, Tanaka M et al (1997) A novel chemotherapy for advanced hepatocellular carcinoma with tumor thrombosis of the main trunk of the portal vein. Cancer 79:1890-1896

8. Okuda K, Ohtsuki T, Obata H et al (1985) Natural history of hepatocellular carcinoma and prognosis in relation to treatment: study of 850 patients. Cancer 56:918-928

9. Pirisi M, Avellini C, Fabris C et al (1998) Portal vein thrombosis in hepatocellular carcinoma: age and sex distribution in an autopsy study. J Cancer Res Clin 124:397-400

10. Masuda T, Beppu T, Ishiko T et al (2008) Intrahepatic dissemination of hepatocellular carcinoma after local ablation therapy. J Hepatobiliary Pancreat Surg 15:589-595

11. Fukuda S, Okuda K, Imamura M et al (2002) Surgical resection combined with chemotherapy for advanced hepatocellular carcinoma with tumor thrombus: report of 19 cases. Surgery 131:300-310

12. Ando E, Tanaka M, Yamashita F et al (2002) Hepatic arterial infusion chemotherapy for advanced hepatocellular carcinoma with portal vein: analysis of 48 cases. Cancer 95:588-595

13. Sakon M, Nagano H, Dono K et al (2002) Combined intraarterial 5-fluorouracil and subcutaneous interferon-alpha therapy for advanced hepatocellular carcinoma with tumor thrombi in the major portal branches. Cancer 94:435-442

14. Beppu T, Ohara C, Yamaguchi Y et al (1991) A new approach to hemoembolization for unresectable hepatocellular carcinoma using aclarubicin microspheres in combination with cisplatin suspended in iodized oil. Cancer 68:2555-2560

15. Beppu T, Ishiko T, Chikamoto A et al (2012) Liver hanging maneuver decreases blood loss and operative time in a right-side hepatectomy. Hepatogastroenterology (in press)

16. Inoue $Y$, Hasegawa $K$, Ishizawa $T$ et al (2009) Is there any difference in survival according to the portal tumor thrombectomy method in patients with hepatocellular carcinoma? Surgery 145:9-19

17. The Liver Cancer Study Group of Japan (1994) Predictive factors for long term prognosis after partial hepatectomy for patients with hepatocellular carcinoma in Japan. Cancer 74:2772-2780

18. Cady B (1983) Natural history of primary and secondary tumors of the liver. Semin Oncol 10:127-134

19. Llovet JM, Bustamante J, Castells A et al (1999) Natural history of untreated nonsurgical hepatocellular carcinoma: rationale for the design and evaluation of therapeutic trials. Hepatology 29:62-67

20. Minagawa M, Makuuchi M (2006) Treatment of hepatocellular carcinoma accompanied by portal vein tumor thrombus. World $\mathrm{J}$ Gastroenterol 21:7561-7567

21. Ikai I, Yamaoka Y, Yamamoto Y et al (1998) Surgical intervention for patients with stage IV-A hepatocellular carcinoma without lymph node metastasis: proposal as a standard therapy. Ann Surg 227:433-439

22. Wu CC, Hsieh SR, Chen JT et al (2000) An appraisal of liver and portal vein resection for hepatocellular carcinoma with tumor thrombi extending to portal bifurcation. Arch Surg 135:1273-1279

23. Le Treut YP, Hardwigsen J, Ananian P et al (2006) Resection of hepatocellular carcinoma with tumor thrombus in the major vasculature: a European case-control series. J Gastrointest Surg $10: 855-862$ 
24. Zhou J, Tang ZY, Wu ZQ et al (2006) Factors influencing survival in hepatocellular carcinoma patients with macroscopic portal vein tumor thrombosis after surgery, with special reference to time dependency: a single-center experience of 381 cases. Hepatogastroenterology 53:275-280

25. Choi KK, Kim SH, Choi SB et al (2011) Portal venous invasion: the single most independent risk factor for immediate postoperative recurrence of hepatocellular carcinoma. J Gastroenterol Hepatol 26:1646-1651

26. Minagawa M, Makuuchi M, Takayama T et al (2001) Selection criteria for hepatectomy in patients with hepatocellular carcinoma and portal vein tumor thrombus. Ann Surg 233:379-384

27. Peng BG, He Q, Li JP et al (2009) Adjuvant transcatheter arterial chemoembolization improves efficacy of hepatectomy for patients with hepatocellular carcinoma and portal vein tumor thrombus. Am J Surg 198:313-318

28. Niguma T, Muira T, Tutui N (2005) Adjuvant arterial infusion chemotherapy after resection of hepatocellular carcinoma with portal thrombosis: a pilot study. J Hepatobilliary Pancreat Surg 12:249-253

29. Huang YH, Wu JC, Lui WY et al (2000) Prospective case-controlled trial of adjuvant chemotherapy after resection of hepatocellular carcinoma. World J Surg 24:551-555. doi:10.1007/s002689910090
30. Ishida K, Hirooka M, Hiraoka A et al (2008) Treatment of hepatocellular carcinoma using arterial chemoembolization with degradable starch microspheres and continuous arterial infusion of 5-fluorouracil. Jpn J Clin Oncol 38:596-603

31. Ikeda K, Saitoh S, Tsubota A et al (1993) Risk factors for tumor recurrence and prognosis after curative resection of hepatocellular carcinoma. Cancer 71:19-25

32. Portolani N, Coniglio A, Ghidoni S et al (2006) Early and late recurrence after liver resection for hepatocellular carcinoma: prognostic and therapeutic implications. Ann Surg 243:229-235

33. Sakon M, Umeshita K, Nagano H et al (2000) Clinical significance of hepatic resection in hepatocellular carcinoma: analysis by disease-free survival curves. Arch Surg 135:1456-1459

34. Yamanaka N, Okamoto E, Fujihara S et al (1992) Do the tumor cells of hepatocellular carcinomas dislodge into the portal venous stream during hepatic resection? Cancer 70:2263-2267

35. Ichida F, Tsuji T, Omata M (1996) New Inuyama classification; new criteria for histological assessment of chronic hepatitis. Int Hepatol Commun 6:112-119 